\title{
Maximal core size in singular graphs
}

\author{
Irene Sciriha* \\ Department of Mathematics, Faculty of Science, University of Malta, Msida, Malta
}

Received 3 September 2009, accepted 5 November 2009, published online 30 November 2009

\begin{abstract}
A graph $G$ is singular of nullity $\eta$ if the nullspace of its adjacency matrix $\mathbf{G}$ has dimension $\eta$. Such a graph contains $\eta$ cores determined by a basis for the nullspace of $\mathbf{G}$. These are induced subgraphs of singular configurations, the latter occurring as induced subgraphs of $G$. We show that there exists a set of $\eta$ distinct vertices representing the singular configurations. We also explore how the nullity controls the size of the singular substructures and characterize those graphs of maximal nullity containing a substructure reaching maximal size.
\end{abstract}

Keywords: Adjacency matrix, nullity, extremal singular graphs, singular configurations, core width.

Math. Subj. Class.: 05C50, 05C60, 05B20.

\section{Introduction}

A graph $G=G(\mathcal{V}, \mathcal{E})$ has vertex set $\mathcal{V}=\mathcal{V}_{G}=\{1,2, \ldots, n\}$ and edge set $\mathcal{E}$ consisting of pairs of vertices. The $\operatorname{order}|G|$ of a graph $G$ is the number $n$ of vertices. The graphs we consider are simple, that is, without multiple edges or loops. The complete graph $K_{n}$ on $n$ vertices has edges between all distinct pairs of vertices.

The graph $G-X$ denotes the graph obtained from $G$ when the set $X$ of vertices and the edges incident to the vertices in $X$ are deleted. The reverse process, starting from $H$ and adding a vertex set $X$ results in $H+X$. Note that $H+X$ is not unique for a particular graph $H$ and set $X$, since it varies with the choice of edges between $X$ and $\mathcal{V}_{H}$ and even with the edges among the vertices of $X$ themselves. If $X=\{v\}$, we write $G-v$ and $G+v$ for $G-X$ and $G+X$ respectively.

The adjacency matrix of a graph $G$, denoted by $\mathbf{G}$, is $\left(a_{i j}\right)$, where $a_{i j}=1$ if $\{i j\}$ is an edge and 0 otherwise. Note that the set of matrices $\{\mathbf{G}\}$ for distinct labellings of the vertices are permutationally similar and therefore a graph $G$ is described completely (up

* This research is supported by the AWRF and project fund R-39 of the University of Malta. Web page http://staff.um.edu.mt/iscil/.

E-mail address: irene.sciriha-aquilina@um.edu.mt (Irene Sciriha) 
to isomorphism) by the corresponding $\mathbf{G}$ for a specific labelling. The spectrum $S p(G)$ of a graph $G$ consists of the collection, with repetitions, of the eigenvalues of $\mathbf{G}$, which are the solutions of the characteristic equation $\operatorname{det}(\lambda \mathbf{I}-\mathbf{G})=0$. The algebraic multiplicity of an eigenvalue is the number of times it is repeated in $\operatorname{Sp}(G)$. The geometric multiplicity is the dimension of the corresponding eigenspace. Since $\mathbf{G}$ is real and symmetric, the two multiplicities for an eigenvalue have a common value. The multiplicity of the eigenvalue zero is referred to as the nullity ${ }^{1}, \eta(G)$, of $G$. The $\operatorname{rank}$ of $G$ is $\operatorname{rank}(\mathbf{G})$, equal to $n-\eta(G)$, a result referred to as the Dimension Theorem.

A graph $G$ on $n$ vertices is said to be singular if $\eta(G)>0$; that is, if there exists $\mathbf{x} \neq \mathbf{0}, \mathbf{x} \in \mathbb{R}^{n}$, such that $\mathbf{G x}=\mathbf{0}$, where each entry of the vector $\mathbf{0}$ is 0 . Since $\mathbf{G}$ satisfies $\mathbf{G} \mathbf{x}=\lambda \mathbf{x}$ for the eigenvalue $\lambda=0$, we call $\mathbf{x}$ a kernel eigenvector of $G$.

This paper is motivated by the question:

How does the nullity control the size of the singular substructures within a graph?

This we address in section 4. To this end, we survey results on substructures in section 2 and on certain invariants of a graph in section 3, including proofs of theorems that facilitate the reasoning of new results, leading to a clarification of the underlying concepts.

\section{Singular graphs}

Let $\mathbf{x} \in \mathbb{R}^{n}, n \geq 3$, be a vector in the nullspace of $G$, which is labelled so that $\mathbf{x}=$ $\left(\mathbf{x}_{F}, \mathbf{0}\right)^{t}$, with each entry of $\mathbf{x}_{F}$ being non-zero. The vertices corresponding to $\mathbf{x}_{F}$ induce a subgraph $F$ whose adjacency matrix is the principal $|F| \times|F|$ submatrix $\mathbf{F}$ of $\mathbf{G}$, satisfying $\mathbf{F x}_{\mathbf{F}}=\mathbf{0}$. We call $\left(F, \mathbf{x}_{F}\right)$, or simply $F$, a core of $G$. If $\mathbf{x}=\mathbf{x}_{F}$, then $G=F$ and $G$ is said to be a core graph. Note that a core of $G$ is a core graph in its own right. Linearly independent kernel eigenvectors determine distinct cores of $G$. The set $\mathrm{CV}$ of core vertices consists of those vertices that lie on some core of $G$. If a vertex does not lie on any core of $G$, then it is said to be core forbidden. A core graph without isolated vertices having nullity one must be connected and is said to be a nut graph. Nut graphs exist for all orders from seven onwards. There are three nut graphs of order seven and none for smaller order [10].
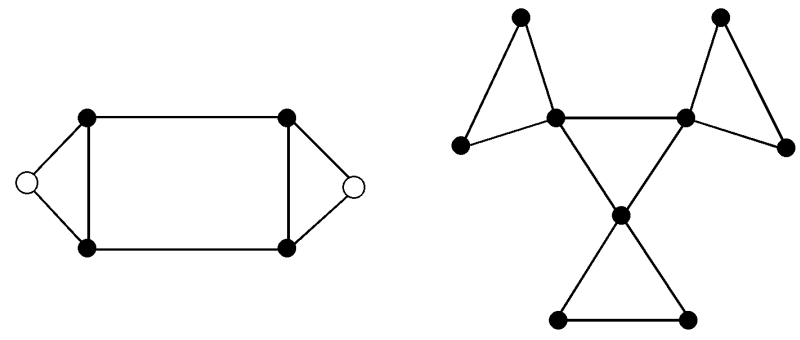

Figure 1: Two singular graphs of nullity one.

Consider the two graphs in Figure 1. The six vertex graph has a nullvector $(1,1,-1$, $-1,0,0)^{t}$ and its core is the four cycle $C_{4}$ (labelled $1,2,3,4$ ), induced by the solid black vertices, while its core-forbidden vertices (labelled 5,6) are white. The nine vertex graph is a nut graph and therefore has no core-forbidden vertices.

\footnotetext{
${ }^{1}$ The term $\operatorname{corank}(\mathbf{G})$ is also used for nullity $(\mathbf{G})$ in the literature.
} 
Since it is the existence of $\mathbf{x}_{F} \in \mathbb{R}^{|F|}$ that determines that a graph is singular, we classify singular graphs according to core-order $|F|$. As shown in Figure 2, there are eight possible cores of order six, three of order five, two of order four and one each of orders three and two.

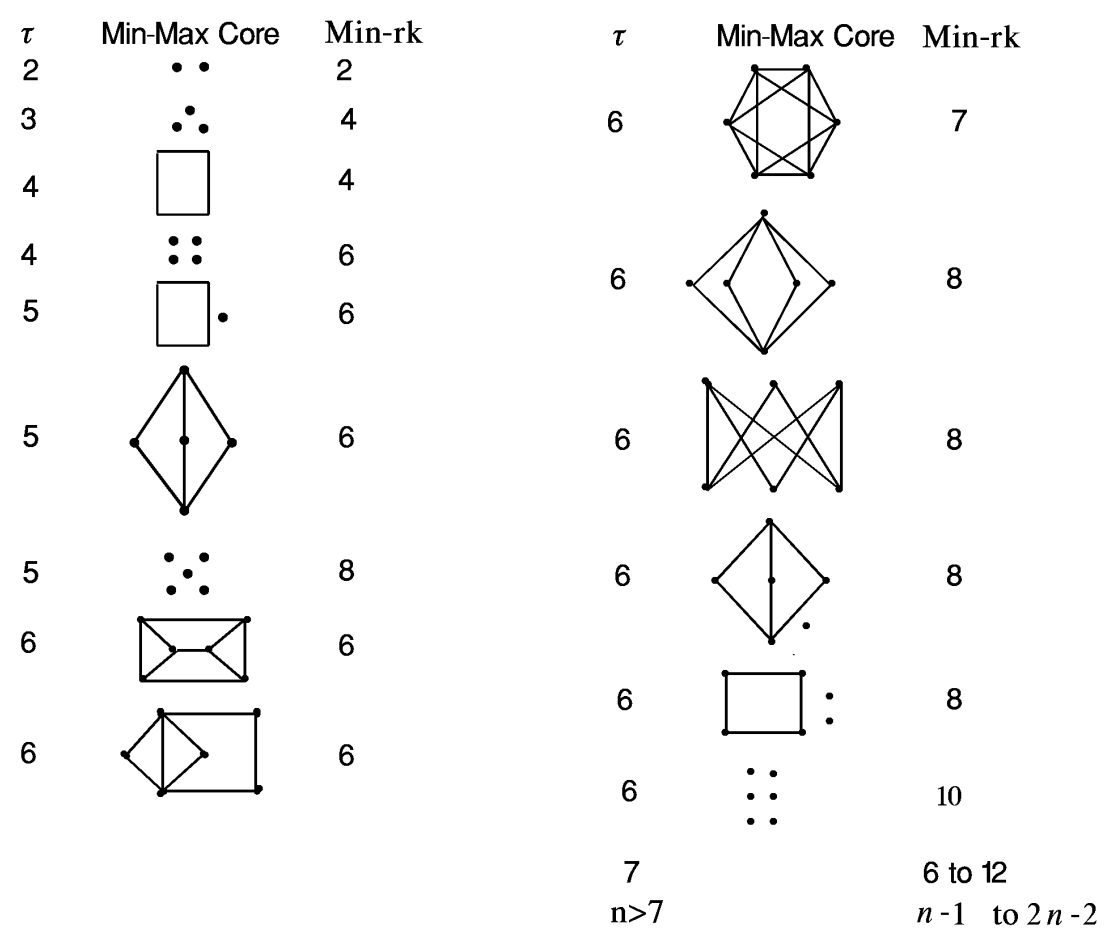

Figure 2: Minimum rank of graphs with core-width $\tau$.

\subsection{Singular configurations}

Cauchy's inequalities for a Hermitian matrix $M$, (also known as the Interlacing Theorem [3]) control the multiplicity of the eigenvalues of principal submatrices relative to those of $M$. Applied to graphs we have:

Theorem 2.1. Interlacing Theorem: Let $G$ be an $n$-vertex graph and $v \in \mathcal{V}$. If the eigenvalues of $G$ are $\lambda_{1}, \lambda_{2}, \ldots, \lambda_{n}$ and those of $G-v$ are $\mu_{1}, \mu_{2}, \ldots, \mu_{n-1}$, both in non-increasing order of magnitude, then $\lambda_{1} \geq \mu_{1} \geq \lambda_{2} \geq \mu_{2} \geq \ldots \geq \mu_{n-1} \geq \lambda_{n}$.

Thus when a vertex is added to a graph, the nullity, or multiplicity of the eigenvalue zero, may change by at most one. Let us first consider a graph of nullity one. Such a graph $G$ with nullspace generator $\mathrm{x}$ has a unique core $F$ as an induced subgraph determined by the vertices corresponding to the non-zero restriction $\mathbf{x}_{F}$ of $\mathbf{x}$. We say the core is $\left(F, \mathbf{x}_{F}\right)$ when $\mathbf{x}_{F}$ needs to be emphasized. By interlacing, to obtain a graph $G$ of nullity one from a core graph $F$ of nullity $\eta$, at least $\eta-1$ vertices are added. Thus a lower bound for the order of a graph $G$ of nullity one, with core $\left(F, \mathbf{x}_{F}\right)$, is $|F|+\eta(F)-1$. 
Definition 2.2. A graph $G,|G| \geq 3$, is a singular configuration (SC), with core $\left(F, \mathbf{x}_{F}\right)$, if it is a singular graph, of nullity one, having $|F|+\eta(F)-1$ vertices, with $F$ as an induced subgraph, satisfying $|F| \geq 2, \mathbf{F} \mathbf{x}_{F}=\mathbf{0}$ and $\mathbf{G}\left(\begin{array}{c}\mathbf{x}_{F} \\ \mathbf{0}\end{array}\right)=\left(\begin{array}{l}\mathbf{0} \\ \mathbf{0}\end{array}\right)$. The vector $\mathbf{x}_{F}$ is said to be the non-zero part of the kernel eigenvector $\left(\begin{array}{c}\mathbf{x}_{F} \\ \mathbf{0}\end{array}\right)$ of $G$.

Note that by interlacing, among singular graphs of nullity one, a singular configuration $G$ has the least number of vertices for its core $\left(F, \mathbf{x}_{F}\right)$. A core graph may be connected as in the cycles $C_{4 k}, k \in \mathbb{Z}^{+}$on $4 k$ vertices or disconnected as in the empty graph $r K_{1}$ consisting of $r$ isolated vertices. An important combinatorial property of core graphs is that they have no pendant edges.

Lemma 2.3. A singular configuration is a connected graph.

Proof. Suppose, for contradiction that a singular configuration $S$ is disconnected. Without loss of generality, $S$ has a connected component $S_{1},\left|S_{1}\right| \geq 3$, having a non-zero kernel eigenvector $\mathbf{x}_{1}$. If the vertices of $S_{1}$ are labelled first, then $\mathbf{S}\left(\begin{array}{c}\mathbf{x}_{1} \\ \mathbf{x}_{2}\end{array}\right)=\left(\begin{array}{l}\mathbf{0} \\ \mathbf{0}\end{array}\right)$ and $\mathbf{S}_{1} \mathbf{x}_{1}=\mathbf{0}$. Note that $S$ cannot have isolated vertices, since these would contribute to the nullity, so that $\eta(S)$ would be more than one. Since $\mathbf{x}_{1} \neq \mathbf{0}$, then $\mathbf{x}_{2}=\mathbf{0}$, otherwise $\left(\begin{array}{c}\mathbf{x}_{1} \\ \mathbf{0}\end{array}\right)$ and $\left(\begin{array}{c}\mathbf{0} \\ \mathbf{x}_{2}\end{array}\right)$ would be linearly independent kernel eigenvectors of $S$, again contradicting that the nullity of $S$ is one. Thus the unique core of $S$ lies in $S_{1}$. Suppose that $\mathrm{x}_{2}$ corresponds to vertices in the non-singular components. But then $S$ does not have a minimum number of vertices for core $F$, a contradiction. Thus $S=S_{1}$.

We now show the relevance of singular configurations. We prove that for nullity-one graphs, of order larger than minimal with respect to core $F$, some singular configuration is an induced subgraph.

Proposition 2.4. A graph $G$ without isolated vertices, of nullity one, with core $\left(F, \mathbf{x}_{F}\right)$, has (at least) one induced subgraph which is a singular configuration with the same core $\left(F, \mathbf{x}_{F}\right)$.

Proof. Let the vertices of $F$ be labelled first. Then the first $|F|$ rows of $\mathbf{G}$ may be partitioned as $(\mathbf{F} \mid \mathbf{C})$ and $(\mathbf{F} \mid \mathbf{C})^{t}\left(\mathbf{x}_{F}\right)=0$. Moreover the rank of $(\mathbf{F} \mid \mathbf{C})$ is $|F|-1$. If $F$ is not itself a SC, its nullity $\eta(F)$ is more than one. There exist $\eta(F)-1$ column vectors of the supplementary matrix $\mathbf{C}$ which are mutually linearly independent and also independent of the columns of $F$. These form the matrix $\mathbf{C}^{\prime}$ such that $\operatorname{rank}\left(\left(\mathbf{F} \mid \mathbf{C}^{\prime}\right)\right)=\operatorname{rank}((\mathbf{F} \mid \mathbf{C}))=$ $|F|-1$. The principal submatrix of $\mathbf{A}(G)$ determined by $\left(\mathbf{A}(F) \mid \mathbf{C}^{\prime}\right)$ is of the form $\mathbf{A}^{\prime}=\left(\begin{array}{cc}\mathbf{A}(F) & \mathbf{C}^{\prime} \\ \left(\mathbf{C}^{\prime}\right)^{\mathbf{t}} & \mathbf{Q}\end{array}\right)$, where $\mathbf{Q}$ is a square matrix. The adjacency matrix $\mathbf{A}^{\prime}$ defines an induced subgraph of $G$, satisfying Definition 2.2. Therefore $G$ is a singular configuration $S\left(F, \mathbf{x}_{F}\right)$. 


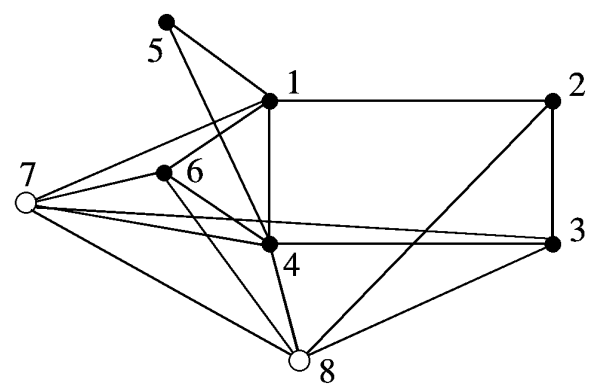

Figure 3: Verices 7 and 8 are core-forbidden.

Example 2.5. A singular configuration $S$ has a unique core $\left(F, \mathbf{x}_{F}\right)$ whose vertices form the full set $\mathrm{CV}$ in $S$. If the set $\mathcal{P}$ of core-forbidden ${ }^{2}$ vertices form an independent subset of the vertices of $S$, then $S$ is said to be a minimal configuration. In fact there are $2^{|\mathcal{P}|}$ singular configurations $\{S\}$ obtained from a particular minimal configuration by adding edges between some pairs of distinct vertices of $\mathcal{P}$ and they all satisfy $\mathbf{S}\left(\begin{array}{c}\mathbf{x}_{F} \\ \mathbf{0}\end{array}\right)=\left(\begin{array}{l}\mathbf{0} \\ \mathbf{0}\end{array}\right)$.

The complete list of minimal configurations of core order up to five may be found in [5].

The singular graph $G$, of nullity one, shown in Figure 3, has only one core $F$ which is the subgraph (of order 6 and nullity 2) induced by the solid black vertices. By Definition 2.2, a singular configuration with core $F$ is expected to have 7 vertices. There are two nonisomorphic singular configurations, $G-7$ and $G-8$, having the same core as $G$. These two distinct induced subgraphs of $G$ are in fact minimal configurations. Moreover, $G$ and its two singular configurations share the same non-zero part $\mathbf{x}_{F}=(1,1,-1,-1,-1,1)^{t}$ of a kernel eigenvector generating their nullspace.

\section{Some new invariants of singular graphs}

The study of singular graphs reveals invariants of a graph. One is the partition of the vertex set $\mathcal{V}(G)$ into the set $\mathrm{CV}$ of vertices lying on some core of $G$ and the set of core-forbidden vertices, $\mathcal{V}(G) \backslash \mathrm{CV}$. We show that this partition does not depend on the choice of basis for the nullspace of $\mathbf{G}$. Therefore $\mathrm{CV}$ is well defined.

Proposition 3.1. The set $\mathrm{CV}$ of core vertices is an invariant of a graph $G$.

Proof. A basis $B$ for the nullspace can be transformed into another, $B^{\prime}$, by linear combinations of the vectors of $B$. However, the union of the collections of the positions of the non-zero entries in the basis vectors is the same for all bases. Thus the partition of the vertex set $\mathcal{V}(G)$ into $\mathrm{CV}$ and core-forbidden vertices, $\mathcal{V}(G) \backslash \mathrm{CV}$, is independent of the basis used for the nullspace.

\footnotetext{
${ }^{2}$ For a graph $G$ of nullity more than one, the periphery with respect to $F$ is the subset of vertices not on the core $F$. For graphs of nullity more than one, the set of core-forbidden vertices is then the set-intersecton of the peripheries over all cores $F$ of $G$.
} 
From proposition 3.1, it follows that the set, $\mathcal{V}(G) \backslash \mathrm{CV}$, of core-forbidden vertices is also an invariant of $G$.

\subsection{Fundamental system of cores}

We now point out properties of subspaces in general that shed light on the structure of a singular graph. For any vector space $W$, let $w t(\mathbf{x})$ denote the weight or number of nonzero entries of the vector $\mathbf{x}$. We adopt the convention to write a basis for a vector space in which the vectors are ordered according to the monotonic non-decreasing sequence of the weights of its vectors.

A maximal set of linearly independent vectors $\mathbf{x}_{1}, \mathbf{x}_{2}, \ldots, \mathbf{x}_{\ell}$, with the smallest weight sum $\sum_{i=1}^{\ell} w t\left(\mathbf{x}_{i}\right)$, are said to form a minimal basis $B_{\min }$ for $W$. We say a basis $B$ for $W$ is reducible if linear combinations of the vectors of $B$ can produce another basis for $W$ with lower weight sum. Thus for any vector space, a basis is not reducible if and only if it is minimal. The monotonic non-decreasing sequence of weights of vectors (weightsequence) in a minimal basis provides an invariant of the vector space, a result that is significant because of its generality to bases of any vector space:

Theorem 3.2. [8] Let $W$ be a q-dimensional subspace of $\mathbb{R}^{n}$. Let $t_{1}$ be the least weight of a non-zero vector in $W$ and let $B_{1}=\left(\mathbf{u}_{1}, \mathbf{u}_{2}, \ldots, \mathbf{u}_{q}\right)$, with weight-sequence $t_{1}, t_{2}, \ldots, t_{q}$, be a basis with minimum weight-sum. If $B_{2}=\left(\mathbf{w}_{1}, \mathbf{w}_{2}, \ldots, \mathbf{w}_{q}\right)$ is another ordered basis for $W$ with weight-sequence $s_{1}, s_{2}, \ldots, s_{q}$, then $\forall i, t_{i} \leq s_{i}$.

Although there may be various possible minimal bases for a vector space, by Proposition 3.2, the sequence of weights of their members is unique for the vector space. The following results are immediate.

Corollary 3.3. A basis for a vector space is not reducible if and only if the monotonic non-decreasing sequence of the weights of its vectors is lexicographically minimal.

Corollary 3.4. The weight-sequence of a minimal basis for a vector space is an invariant of the vector space.

The vertex space for a $n$-vertex graph $G$ is considered to be $\mathbb{R}^{n}$ and the nullspace $\operatorname{ker}(\mathbf{G})$, is a subspace of dimension $\eta$. Note that a minimal basis $B_{\text {min }}$ for $\operatorname{ker}(\mathbf{G})$ determines a fundamental system of cores of $G$. We now apply Corollary 3.4 to singular graphs to obtain another invariant associated with the nullspace.

Proposition 3.5. The monotonic non-decreasing sequence of core-orders in a fundamental system of cores is a graph invariant.

Remark 3.6. The following result proved in [7], provides a necessary condition, in terms of admissible subgraphs, for a graph to be of a specific nullity $\eta$.

Proposition 3.7. Let $H$ be a singular graph, without isolated vertices, having nullity $\eta$. There exist $\eta \mathrm{SC}$ s as induced subgraphs of $H$ whose cores form a fundamental system of cores of $H$.

\subsection{Increasing the nullity of a graph}

Proposition 3.7 provides a necessary condition for a graph to be of nullity $\eta$. The following proposition, which we shall use repeatedly in what follows, gives a necessary and sufficient condition to increase the number of core vertices in a graph. 
Proposition 3.8. The nullity increases with the addition of a vertex $v$ to a graph $G$ forming a connected graph $G+v$ if and only if $v$ is a core vertex of $(G+v)$.

Proof. Suppose, for contradiction, that $v$ does not lie on any core of the resulting graph $G+v$ but $\eta(G)<\eta(G+v)$. Then all the cores of $G+v$ lie in $G$. But then, interlacing demands that $\eta(G) \geq \eta(G+v)$, a contradiction.

Conversely, let $v$ be a core vertex of $(G+v)$. Then there exists a kernel eigenvector $\mathbf{x}$ with a non-zero entry corresponding to $v$. Let $\mathbf{M}$ be the $k \times n$ matrix whose rows are the $k$ vectors of a basis for the nullspace of $G+v$, labelled so that the first row of $\mathbf{M}$ is $\mathbf{x}$ and the first column corresponds to $v$. By row-reducing $\mathbf{M}$ to echelon form, with all entries in the columns above and below a leading 1 (or pivot) being zero, a matrix $\mathbf{M}^{\prime}$ is produced, whose rows give a new basis $B \mathcal{Y}$ for the nullspace of $G+v$, determining a system $\mathcal{Y}$ of cores. The first row determines the only core $F$ in this system with vertex $v$. Deleting $v$ has the effect of destroying $F$ while retaining all the other cores in $\mathcal{Y}$. Thus $\eta(G) \geq \eta(G+v)-1$.

Moreover, if $\eta(G)>\eta(G+v)-1$, then there exists a kernel eigenvector $\mathbf{z}$ of $G$ which is linearly independent of those determined by $\mathcal{Y}$. This kernel eigenvector becomes an additional kernel eigenvector of $G+v$ by adding a zero as a first entry; but then the $k+1$ vectors in $B \mathcal{Y} \cup\{\mathbf{z}\}$ are linearly independent in the $k$-dimensional nullspace of $G+v$, a contradiction. Hence $\eta(G)=\eta(G+v)-1$.

We now show that if a graph $G$ of rank $r$ is labelled so that the first $r$ rows of $\mathbf{G}$ are linearly independent, then $\mathbf{G}$ has a simple block form.

Proposition 3.9. Let the graph $G$ be of order $n$ and rank $r$, with adjacency matrix $\mathbf{G}$. Let the first $r$ rows of $\mathbf{G}$ be linearly independent vectors. Then there exist a non-singular $r \times r$ matrix $B$ and $a r \times(n-r)$ matrix $\mathbf{Y}$ such that

$\mathbf{A}(G)=\left(\begin{array}{cc}\mathbf{B} & \mathbf{B Y} \\ \mathbf{Y}^{t} \mathbf{B} & \mathbf{Y}^{t} \mathbf{B} \mathbf{Y}\end{array}\right)$.

Proof. The first $r$ rows of $\mathbf{G}$ form a submatrix $\mathbf{T}$ of the adjacency matrix $\mathbf{G}$ and represent a maximal set of linearly independent row vectors of $\mathbf{G}$. Each of the $\eta(G)$ row vectors $R_{j}, j>r$, is linearly dependent on a subset of the first $r$ row vectors of $\mathbf{G}$. The adjacency matrix $\mathbf{G}$, of rank $r$ and nullity $\eta$, can be expressed as $\left(\begin{array}{c}\mathbf{T} \\ \mathbf{Y}^{\mathbf{t}} \mathbf{T}\end{array}\right)$ for a $r \times \eta$ matrix $\mathbf{Y}$. To see this, note that each linear relation between $R_{j}, j>r$ and the rows of $\mathbf{T}$ corresponds to a kernel eigenvector in the nullspace of $\mathbf{G}$. Since each of these $\eta(G)$ kernel eigenvectors corresponds to a core with a unique vertex $v_{j}$ (described by row vector $R_{j}, j>r$ ), these $\eta(G)$ kernel eigenvectors are linearly independent and so their first $r$ entries form the columns of $\mathbf{Y}$. Because of the symmetry of $\mathbf{G}$, the block $\mathbf{T}$ can be expressed as $(\mathbf{B} \mathbf{B Y})$, where $\mathbf{B}$ is a $r \times r$ non-singular matrix. The result now follows immediately from the symmetry of $\mathbf{G}$.

Note that in general there are different choices of the first $r$ rows. Also, each of the last $(n-r)$ vertices in this labelling of $G$ lies on a core and are said to form a singular configuration vertex- representation denoted by $\mathcal{R}$. Thus $|\mathcal{R}|=n-r=\eta$, by the Dimension Theorem. The kernel eigenvectors, forming a basis for the nullspace of $\mathbf{G}$, define a system $\mathcal{Y}$ of $\eta$ distinct cores that correspond to $\eta$ singular configurations found as induced subgraphs of $G$. There is a one-one correspondence between $\mathcal{Y}$ and $\mathcal{R}$. The concept of the singular-configuration-vertex-representation has been used in an ad hoc manner in the literature and more formally in this paper. 
We can also identify a singular-configuration-vertex-representation as the set of vertices corresponding to the pivots in the rows of matrix $\mathbf{M}^{\prime}$ in the proof of Proposition 3.8. These define a vertex-representation of a fundamental system $\mathcal{Y}$ of $\eta(G)$ distinct cores. By Proposition 3.8, deleting a vertex $v$ in a singular-configuration-vertex-representation reduces the nullity by one and destroys a core in $\mathcal{Y}$ that has vertex $v$.

Note that any collection of $h$ vectors in $B_{\min }$ collectively cover at least $h$ core-vertices of $G$. So the existence of a singular-configuration-vertex-representation is also guaranteed by Hall's Theorem (also known as the Marriage Problem).

Retaining the labelling of Proposition 3.9, there exist $\eta(G)$ singular configurations (with distinct spanning minimal configurations) which are induced subgraphs of $G$ such that each singular configuration corresponds to a unique vertex $j, r<j \leq n$. By scaling the corresponding kernel eigenvectors so that the last non-zero entry is 1 , the following result is immediate.

Corollary 3.10. Let $G$ be of order $n$ and rank $r$, with adjacency matrix $\mathbf{G}$. Let the first $r$ rows of $\mathbf{G}$ be linearly independent vectors. There exists a matrix $\mathbf{M}^{t}=\left(\begin{array}{c}\mathbf{K} \\ \mathbf{I}\end{array}\right)$ whose columns are the vectors of a basis for the nullspace of $G$, where the order of identity matrix I is $n-r$.

Note that if $\mathbf{G}=\left(\begin{array}{cc}\mathbf{B} & \mathbf{E} \\ \mathbf{E}^{\mathbf{t}} & \mathbf{U}\end{array}\right)$ has rank $r$ and $\mathbf{B}$ is a non-singular $r \times r$ matrix, then $\mathbf{K}=\mathbf{B}^{-1} \mathbf{E}=-\mathbf{Y}$ in Proposition 3.9.

\section{Nullity and core width}

Remark 4.1. Henceforth we shall refer to a minimal basis $B_{\min }$ for $\operatorname{ker}(\mathrm{G})$ as $\left(\mathbf{u}_{1}, \mathbf{u}_{2}, \ldots\right.$, $\left.\mathbf{u}_{\eta}\right)$. The integers $w t\left(\mathbf{u}_{1}\right)$ and $w t\left(\mathbf{u}_{\eta}\right)$ are extremal values and have been referred to as the graph singularity $\kappa$ in [2,9] and core-width $\tau$ in [6], respectively. We focus on the zero, non-zero pattern of the entries of the vectors in $\operatorname{ker}(\mathbf{G})$, to show that the nullity controls the core width.

The zero eigenvalue equation, $\mathbf{G x}=\mathbf{0}$, stipulates that the sum of the entries of $\mathbf{x}$ corresponding to neighbours of any vertex in $G$ is zero. This is also known as the Zero Sum Rule [9] which leads to a generic kernel eigenvector $\mathbf{x}_{g e n}$ in terms of $\eta(G)$ independent parameters. One way in which to obtain a basis of $\eta(G)$ kernel eigenvectors is to set a parameter in turn equal to one and the remaining parameters equal to zero. If $\eta$ non-zero entries of $\mathbf{x}_{g e n}$, chosen so that they are collectively functions of all the $\eta$ parameters, are set to zero, then $\mathbf{x}_{\text {gen }}$ is forced to vanish. This concept, which has been used in the theory of singular graphs [4], will be expanded upon in this section.

The Lemma that follows appears in [1] for graphs with weighted edges. We give a new proof for the $(0-1)$-adjacency matrix, emphasising the role of the core vertices in a singular graph. The result will enable us to relate the core-width $\tau$ to the nullity $\eta$, while the proof helps to shed more light on the graph structure.

Lemma 4.2. If $\eta(G)>k \geq 1$, then there exists $\mathbf{x} \in k e r(G)$, such that $\mathbf{x}$ has zero entries in any $k$ specified positions.

Proof. Let $v$ be a core-forbidden vertex. Then any kernel eigenvector has a zero entry in that position. To prove this lemma, then, we need to show that there exists a kernel eigenvector with zeros in any $k$ positions corresponding to core vertices. 
Let the $k$ chosen core vertices be labelled first, followed by the rest of the core vertices and ending with the core-forbidden vertices. Let $\mathbf{M}$ be the matrix whose rows are the vectors of a basis for the nullspace of $G$, ordered so that the $i$ th entry in the $i$ th row vector is non-zero. The only non-zero entries lie in the first $|\mathrm{CV}|$ columns. Row-reducing $\mathbf{M}$ to echelon form, with all entries in the columns above and below a leading 1 being zero, produces a matrix $\mathbf{M}^{\prime}$, whose rows give a new basis for the nullspace of $G$. Thus each row of $\mathbf{M}^{\prime}$ is non-zero. Furthermore, the pivots in the rows from the $(k+1)$ th up to the last row have at least $k$ zero entries preceding them. Thus the kernel eigenvectors represented by each of the last $\eta(G)-k$ rows of $\mathbf{M}^{\prime}$ satisfy the conditions of the required result.

Lemma 4.2 guarantees a kernel eigenvector with zero entries in any $\eta(G)-1$ specified positions. In the proof, the rows of $\mathbf{M}^{\prime}$ form a basis that can determine the minimum weight sequence and one of a possible number of minimal bases. The determination of the minimum rank of a $(0-1)$-adjacency matrix, as $\tau$ varies, is regarded as an extremal problem. The minimum rank for various values of $\tau$ is given in Figure 2. The result that follows holds for minimal bases.

Proposition 4.3. If $\mathrm{x} \in B_{\text {min }}$, then $\mathrm{x}$ has at least $\eta(G)-1$ zero entries.

Proof. There exists a basis $B$ of kernel eigenvectors which are the rows of the $\eta \times n$ matrix $\mathbf{M}^{\prime}$, with all entries in the columns above and below a pivot being zero. Recall that the set of vertices corresponding to the pivots represents a singular-configuration-vertexrepresentation. Thus any row of $\mathbf{M}^{\prime}$ has at least $|\eta(G)|-1$ zero entries corresponding to all the other rows of $\mathbf{M}^{\prime}$. By Theorem 3.2, the weight-sequence of $B_{\min }$ is entry-wise less than that of $B$. Thus if $\mathbf{x} \in B_{m i n}$, then $\mathbf{x}$ has at least $\eta(G)-1$ zero entries.

By Proposition 3.2, among all (ordered) bases for the nullspace of $\mathbf{G}$, a minimal basis has the maximum number of zeros in each vector in turn. The minimum number of zeros in the vectors of a minimal basis is used to establish a method of obtaining an upperbound for the nullity.

Proposition 4.4. If the number of zero entries in a vector $\mathrm{x} \in B_{\min }$ is less than $k$, then $\eta(G) \leq k$.

Proof. By Proposition 4.3, all the vectors in $B_{\min }$ have $\eta-1$ or more zero entries so that $k-1 \geq \eta-1$, as required.

Note that Proposition 4.4 can also be proved by using Lemma 4.2 directly. This guarantees a vector $\mathbf{y}$ in the nullspace with at least $k$ zeros if $\eta(G)>k$. So suppose that $\eta(G)>k$ and the number of zero entries in each $\mathbf{x} \in B_{\min }$ is $k-1$ or less. Since $\mathbf{y}$ is a linear combination of the vectors in $B_{\text {min }}$, by Theorem 3.2, it cannot have more than $k-1$ zero entries, contradicting the necessary condition of Lemma 4.2. Hence $\eta(G) \leq k$.

To determine the minimum number of zeros over all $\mathrm{x} \in B_{\min }$, it suffices to determine the core-width $\tau$, an invariant of the graph. Figure 2 shows all the cores of order up to six and the minimum rank of graphs with a min-max core-order (or core-width) $\tau$.

We now show that $\tau$ and $\eta$ exert mutual control.

Proposition 4.5. For a graph $G$ on $n$ vertices of nullity $\eta$ and core width $\tau, \tau+\eta \leq n+1$. 
Proof. By definition of core-width $\tau$, there exists $\mathbf{y} \in B_{\min }$ having $\tau$ non-zero entries. If $z$ denotes the number of zero entries in $\mathbf{y}$, then $\tau+z=n$. By Proposition 4.3, $z \geq \eta(G)-1$. Thus $\tau+\eta(G)-1 \leq n$, as required.

We may ask what the threshold order of a singular graph $G$ needs to be for a particular core $F$ to lie in a fundamental system $\mathcal{F}$ of cores.

Corollary 4.6. A singular graph $G$ of nullity $\eta$ cannot have a core $F_{t}$ of order $t$ in $\mathcal{F}$ if $t>n+1-\eta$.

Definition 4.7. Let the order of a singular graph $G$ be $n$. If the nullity is $\eta$ and the core width is $\tau$, then $G$ is said to be extremal singular if $\eta+\tau$ reaches $n+1$.

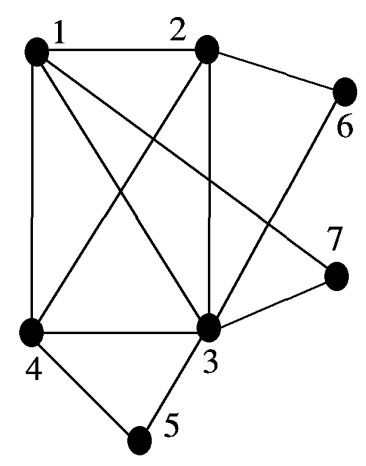

Nut graph on 7 vertices

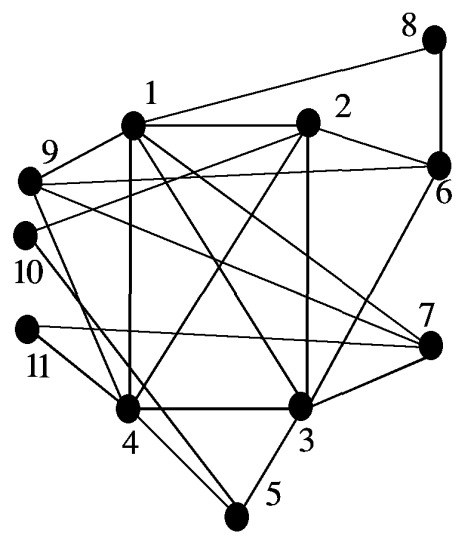

Extremal Singular: $\kappa=3, \tau=7$ Core-Order Sequence: $3,3,3,4,7$

Figure 4: The nut graph $7 \mathrm{~b}$ and a extremal singular graph of nullity five.

To determine for which graphs $\tau+\eta$ reaches the upper bound $n+1$, we need to consider as large a core width $\tau$ as is possible, for a given core-order $n$ and nullity $\eta$. Figure 4 shows a core graph which is extremal singular with the nut graph $N_{7 b}$ in a fundamental system of cores.

Proposition 4.8. A graph $G$ is extremal singular of nullity $\eta$, if and only if it is a core graph and the largest core in a fundamental system is a nut graph $N$ and there are exactly $\eta-1$ vertices of $G$ not on $N$.

Proof. Let $\tau+\eta=n+1$ and $\mathbf{x} \in B_{\text {min }}$ have $\tau$ non-zero entries representing core $F_{\tau}$. If $z$ denotes the number of zero entries in $\mathbf{x}$, then $\tau+z=n$ and $z$ needs to be $\eta-1$. As in the proof of Proposition 4.3, there exists a subset $L$ consisting of $\eta-1$ vertices in a singularconfiguration-vertex-representation representing the cores for $B_{\min }$ other than $F_{\tau}$, such that at the vertex positions of $L$, the entries of $\mathrm{x}$ are zero, but the entries of the other $\eta-1$ vectors in $B_{\min }$ are collectively non-zero. Then corresponding to each vertex of $G$, there is a kernel eigenvector with a non-zero entry in the associated position. Hence each vertex 
of $G$ is a core vertex. Now deleting $L$ destroys exactly $\eta-1$ cores of $G$ leaving a subgraph $H$ of $G$ of order $\tau$ with core $F_{\tau}$ whose kernel eigenvector has $\tau$ non-zero entries. Thus $G$ is a core graph and $H$ is a nut graph whose kernel eigenvector is the non-zero part of $\mathbf{x}$.

Conversely, if in a core graph $G$, the core corresponding to the core-width $\tau$ is a nut graph $N$, then $\tau+\eta(N)=|N|+1$. By Proposition 3.8, as each of the $\eta-1$ vertices is added to $N$ to produce $G$, the graph proceeds through a series of core graphs. Both sides of the equation increases by one at each stage. Thus $\tau+\eta(G)=|G|+1$.

Proposition 4.9. A graph $G$ is extremal singular of nullity one if and only if $G$ is a nut graph.

Proof. Recall that a nut graph is a core graph of nullity one. Thus $\tau=n$ and $\tau+\eta$ reaches the upper bound. Now for a graph with nullity one which is not a nut graph, there exist core-forbidden vertices so that $\tau<n$.

Thus a nut graph of order $n_{1}$ is extremal singular and may be grown into larger extremal singular graphs with core width $n_{1}$.

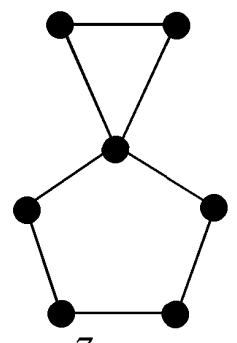

7 - nut

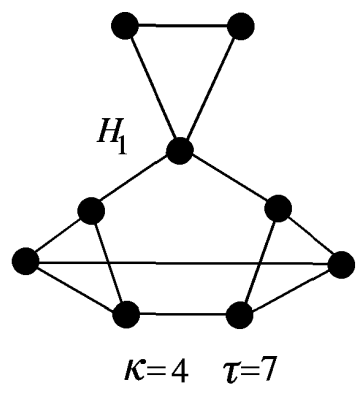

Add vertices to 7 - nut

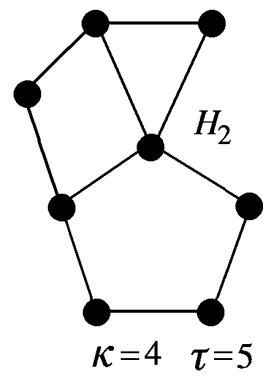

$\kappa=4 \quad \tau=5$

Figure 5: Non-extremal core graphs $H_{1}$ and $H_{2}$ of nullity two - with the nut graph $N_{7 a}$ as an induced subgraph.

When starting from a nut graph $\left(N, \mathbf{x}_{N}\right)$, to construct a singular graph of nullity $\eta$, in such a way that the nullity increases with each vertex addition, the vector $\mathbf{x}_{N}$ need not remain in a minimal basis. Indeed, let us start with a nut graph $N$ and add vertices to produce a core graph. If with each vertex added, a core graph is created while $\left(N, \mathbf{x}_{N}\right)$ is preserved, then the nullity increases by one with each vertex addition and the equality $|N|+\eta(G)=n+1$ holds. If the core-width $\tau(G)=|N|$, then $G$ is extremal singular. However, there are two other possibilities that might occur in the process of vertex addition until the $n$-vertex core graph $G$ is produced. With some vertex addition, either a core is not created or the core $\left(N, \mathbf{x}_{N}\right)$ is not preserved in a basis $B_{\min }$ for the enlarged graph $G$. In the former case the nullity does not reach the maximum possible for $n$ and $\tau$, whereas in the latter case $\mathbf{x}_{N}$ does not remain the non-zero part of a vector in a basis $B_{\min }$ for the nullspace of the adjacency matrix of the enlarged graph obtained. If either of these cases occurs, even though the nut graph $N$ remains an induced subgraph and $\mathbf{x}_{N}$ is still a kernel eigenvector of the enlarged graph, $\tau+\eta<n+1$, and the core graph obtained is not extremal singular. The graphs in Figure 5 illustrate these two cases. The nine-vertex 
graph $H_{1}$ has $\kappa=4$ and $\tau=7$, with $F_{\tau}$ being the nut graph $N_{7 a}$ (the smallest nut graph possible [10]). The nullity is two, however, not three as required for $\tau+\eta=n+1$, because the nullity did not increase on adding the first vertex to the nut graph $N_{7 a}$. Note that the eight vertex graph $\mathrm{H}_{2}$, in Figure 5, has nullity two as well. Although the nut graph $N_{7 a}$ is an induced subgraph and a core of $H_{2}, \tau$ is not preserved. Thus the nut graph $N_{7 a}$ fails to remain in a Fundamental System of cores (determined by a $B_{\min }\left(H_{2}\right)$ ) after the eighth vertex is added.

Proposition 4.10. Let $G$ be a singular graph of nullity $\eta$, having a fundamental system $\mathcal{F}$ of cores. Then $\max _{F \in \mathcal{F}}(|F|+\eta(F))+\eta(G) \leq n+2$.

Proof. Let $F \in \mathcal{F}$ have kernel eigenvector $\mathbf{x}_{F}$. By Proposition 3.7, there exists a singular configuration $H$ of order $|F|+\eta(F)-1$ with core $\left(F, \mathbf{x}_{F}\right)$ as an induced subgraph of $G$. By interlacing, $G$ has at least $|H|+\eta(G)-1$ vertices. Hence $n \geq|F|+\eta(F)+\eta(G)-2$ for all $F \in \mathcal{F}$.

A core in a fundamental system may be a nut graph in which case it is both a core of $G$ and an induced singular configuration of $G$. If $G$ is extremal singular, then by Proposition 4.8, a core of maximum order $\tau$ in $\mathcal{F}$ is a nut graph. Recall that any core $F$, in a fundamental system $\mathcal{F}$ of cores of a graph $G$ is an induced subgraph of a singular configuration $H$ which is in turn an induced subgraph of $G$. Now by definition, $|F| \leq \tau$ but is $|H| \leq \tau$ ? This is extremely pertinent when a core in a fundamental system $\mathcal{F}$ of cores of a graph $G$ is $r K_{1}$. In this case, the order, $2|F|-1$, of $H$ is relatively large when compared to $|F|$, in contrast with the case when $F$ is a nut graph and $|H|=|F|$.

Lemma 4.11. Let $G$ be an extremal singular graph with core width $\tau$ and nullity $\eta$. Let $F$ be a core in a fundamental system of cores of $G$. Then $\tau \geq|F|+\eta(F)-1$.

Proof. Since $G$ is extremal singular, $\eta(G)+\tau=n+1$. By Proposition 4.10, $|F|+\eta(F)-$ $1 \leq n+1-\eta(G)=\tau$.

Proposition 4.12. Let $G$ be an extremal singular graph of nullity $\eta$ and core-width $\tau$. Let $F$ be a core having a fundamental system $\mathcal{F}$ of cores. If $H$ is an induced subgraph of $G$ which is a singular configuration with core $F$, then $|H| \leq \tau$.

Proof. Let $H$ be a singular configuration which is an induced subgraph of $G$ corresponding to the core $F$. By Lemma 4.11, $|H|=|F|+\eta(F)-1 \leq \tau$.

Example 4.13. The singular configurations of the extremal singular graph of Figure 4, associated with the cores $3 K_{1}, 3 K_{1}, 3 K_{1}, C_{4}$, and the nut graph $N_{7 a}$ in a fundamental system, have order 5, 5, 5, 5, 7 respectively, supporting Proposition 4.12.

The graphs $H_{1}$ and $H_{2}$ of Figure 5 are not extremal singular. The graph $H_{1}$ has coreorder sequence $\{4,7\}$ corresponding to cores $C_{4}$ and the nut graph $N_{7 a}$, associated to singular configurations of order 5 and 7 respectively. The graph $H_{2}$ has core-order sequence $\{4,5\}$ corresponding to cores $4 K_{1}$ and $C_{4} \dot{\cup} K_{1}$, associated to singular configurations of order 5 and 7 respectively.

Corollary 4.14. If core $r K_{1}$ is in fundamental system of cores of an extremal singular graph, then $r \leq\left\lceil\frac{\tau}{2}\right\rceil$. 
Proof. A singular configuration $H$ with core $r K_{1}$ has $(2 r-1)$ vertices. Then $|H|=2 r-1$. By Proposition 4.12, $|H| \leq \tau$. Hence the integer $r \leq \frac{\tau+1}{2}$.

\section{Conclusion}

The symmetry of the $(0-1)$-adjacency matrix $\mathbf{G}$ of rank $r$ enables its representation as a block matrix with a maximally non-singular $(r \times r)$-principal submatrix of $\mathbf{G}$. The nullspace of $\mathbf{G}$ can be expressed in terms of submatrices of this representation.

The presence of singular configurations as induced subgraphs in a graph is a necessary condition for a graph to be singular. In a graph $G$ of nullity $\eta$, we showed that there is a vertex representation $\mathcal{R}$ consisting of $\eta$ vertices corresponding to $\eta$ singular configurations which are induced subgraphs of $G$. The choice of the vertices in $\mathcal{R}$, which are core vertices, is not unique. A graph may have core-forbidden vertices that do not lie on any core of $G$. Deletion of a core-forbidden vertex either increases the nullity or leaves it unchanged.

The core-width $\tau$ (maximum weight of the vectors in a minimal basis for the nullspace of $G$ ) is an invariant of $G$ and was shown to have extremal properties tied with the nullity $\eta$ in a $n$-vertex graph. Among all graphs on $n$ vertices, $\tau+\eta$ reaches a maximum in extremal singular graphs. Not only are the core-orders in a fundamental system $\mathcal{F}$ of cores bounded above by $\tau$ but in extremal singular graphs, the orders of the singular configurations corresponding to the cores of $\mathcal{F}$ are also bounded above by $\tau$.

\section{References}

[1] AIM Minimum Rank - Special Graphs Work Group, Zero forcing sets and the minimum rank of graphs, Linear Algebra Appl. 428 (2008), 1628-1648.

[2] M. Brown, J. W. Kennedy and B. Servatius, Graph singularity, Graph Theory Notes of New York, The New York Academy of Sciences 25 (1993), 23-32.

[3] A. J. Schwenk and R. J. Wilson, On the eigenvalues of a graph, in: L. W. Beineke and R. J. Wilson (eds.), Selected Topics in Graph Theory, Academic Press, London, 1978, 307-336.

[4] I. Sciriha, On the coefficient of $\lambda$ in the characteristic polynomial of singular graphs, Util. Math. 52 (1997), 97-111.

[5] I. Sciriha, On the construction of graphs of nullity one, Discrete Math. 181 (1998), 193-211.

[6] I. Sciriha, On the rank of graphs, in: Y. Alavi, D. R. Lick and A. Schwenk (eds.), Combinatorics, graph theory and algorithms 2, New Issues Press, Kalamazoo, Michigan, 1999, 769778.

[7] I. Sciriha, A characterization of singular graphs, Electron. J. Linear Algebra 16 (2007), 451462.

[8] I. Sciriha, S. Fiorini and J. Lauri, A minimal basis for a vector space, Graph Theory Notes of New York, The New York Academy of Sciences 31 (1996), 21-24.

[9] I. Sciriha and I. Gutman, Graphs with maximum singularity, Graph Theory Notes of New York, The New York Academy of Sciences 30 (1996), 17-20.

[10] I. Sciriha and I. Gutman, Nut graphs: maximally extending cores, Util. Math. 54 (1998), $257-$ 272. 